\section{Letters to the Editor.}

[The Editor does not hold himself responsible for opinions expressed by his correspondents. Neither can he undertake to return, nor to correspond with the writers of, rejected manuscripts intended for this or any other part of NATURE. No notice is taken of anonymous communications.]

Propagation of Magnetic Disturbances along Wires.

IT is well known that the magnetisation of a ferromagnetic material changes in small steps (Barkhausen effect). We have shown ${ }^{1}$ for a variety of materials that the sizes of these steps, or sudden changes in magnetisation, are independent of the sizes of the crystals of which the material is composed, and

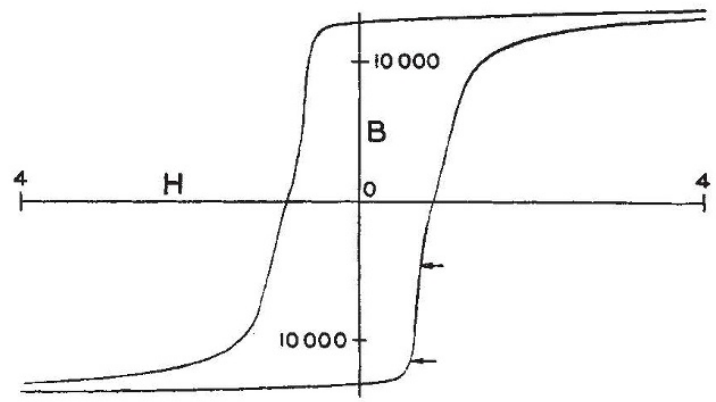

FiG. 1.--Hysteresis loop of Armco iron, maximum field strength 10 gauss.

correspond to the sudden reversal of the magnetic moment of a volume of material of the order of $10^{-8}$ or $10^{-9} \mathrm{~cm}^{3}$.

We now find that in many materials these small steps in magnetisation occur in groups of such size as to be detectable with a sensitive galvanometer. This effect for iron is illustrated in the accompanying figures. Fig. 1 shows a hysteresis loop of Armco iron recorded photographically with the fluxmeter recently described by Haworth. ${ }^{2}$ No discontinuities are apparent here. Fig. 2 represents the portion of the loop lying between the arrows shown in Fig. 1,

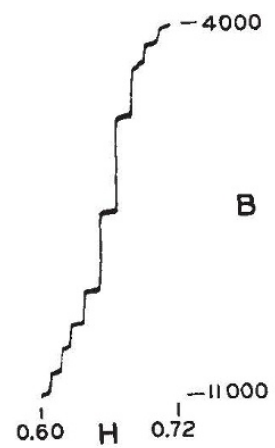

Fra. 2.--Portion of loop of

Fig. 1, recorded slowly. recorded more slowly with greater fluxmeter sensitivity, and shows the large steps under consideration. These steps appear only when the field is changed slowly; this is because it takes the magnetisation several tenths of a second to increase in any one step, and if during that time the field strength increased enough to set off the next change in mag. netisation, the steps will not be separable. While taking Fig. 2 the field strength was changed $0 \cdot 2$ gauss per minute.

That each such step in mag. netisation is composed of a great many separate smaller changes is shown in Fig. 3, where the whole record refers to the change in magnetisation occurring in one of the steps shown in Fig. 2. Each sharp point of the record indicates one of the small steps or discontinuities discovered by Barkhausen. The record was made with the usual arrangement ${ }^{3}$ of search coil, amplifier, and oscillograph, used with a slowly and uniformly changing magnetic field strength.

Separate tests have shown us that the change in magnetisation corresponding to one of the single steps of Fig. 2 occurs throughout all sections of the sample, except near the ends, where the field is far from uniform. This fact suggested that the magnetic disturbance was transmitted from one part of the sample to another by mechanical vibrations produced by the sudden change in length accompanying the change in magnetisation (magnetostriction). The other way in which the disturbance might be propagated is by magnetic influence alone. According to the latter idea, the increase in magnetisation occurring at one point increases the field strength and consequently the magnetisation at nearby points. To distinguish between these twio methods of propagation, an experiment was made as follows: The large steplike changes were made to occur over almost the whole length of the iron wire as before, but were prevented from occurring in a length of two centimetres in the middle by applying a field there in the opposite direction. The sudden changes in the two halves of the wire were then found to be completely independent of each other, or incoherent, whereas with no back field in the middle they were always coherent. Since elastic waves pass unhindered through the middle portion, and experiment showed that no change in magnetisation occurred here, it is concluded that purely magnetic processes are responsible for keeping the disturbance going once it is started.

We have examined similar disturbances in annealed iron wires so fine as $0.0017 \mathrm{~cm}$. in diameter and found that the changes in magnetisation are in much larger steps, a single step accounting for almost the whole change from saturation in one direction to saturation in the other. These recall the results in hard drawn wires under tension and torsion described by Preisach and by Sixtus and Tonks. ${ }^{5}$ In our own experiments, when the diameter is less than about $0.01 \mathrm{~cm}$., the

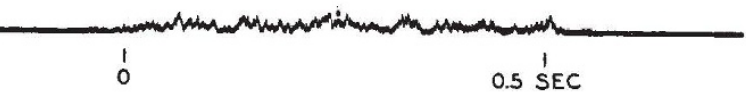

IG. 3.-Oscillograph record of one of the single steps of Fig. 2, ordinates proportional to time rate of change of magnetisation.

separate small Barkhausen discontinuities cannot be detected even with an oscillograph recording frequencies up to 4000 cycles per second. The whole change occurs in one step and travels along the wire with a speed inversely proportional to the square of the diameter of the wire. This also suggests that the magnetic field strength controls the propagation, since the rate of decay of eddy-currents varies inversely as the square of the diameter.

R. M. Bozorth.

Bell Telephone Laboratories, Inc.,

$$
\text { J. F. Dillinger. }
$$

New York, N.Y. April 6.

I Phys. Rev. (2), 35, 733-52; 1930

2 Bell Sys. Tech. Jour., 10, 20-32;1931.

3 Loc cit. 1. The oseillograph was recently described by A. M. Curtis, Bell Record, 8, 580; 1930

${ }^{4}$ Ann. der Phys. (5), 3, 737-99; 1929

5 Phys. Rev. (2), 35, 144-1; 1930.

\section{The Earth's Thermal History.}

DR. J. H. J. Pooce has kindly pointed out to me that the reference to the adiabatic gradient of temperature in my previous letter ${ }^{1}$ is capable of being understood to mean that he believes that the adiabatic gradient in a liquid heated below would not be maintained. This was not intended; the maintenance of the adiabatic gradient by convection currents (or rather, of one exceeding it by the trifling amount

No. 3212, VoL. 127] 